\title{
Editorial for June 2021 issue
}

\author{
S. V. Raghavan ${ }^{1}$
}

Published online: 19 August 2021

(C) CSI Publications 2021

The June 2021 issue in your hands carries a series of interesting articles about FinTech. As Information Technology evolved, one of the domains that benefitted most was the Financial Transaction. In finance related domain, many activities such as banking, insurance, securities, micro-financing, and electronic commerce befitted immensely benefitted and scaled in volume and geographical coverage. Alongside many issues related to jurisdiction, responsibility resting, dispute resolution, scenario re-creations, etc. gained importance. The recognition that policies relating to financial activities across domains of activity and sovereign boundaries started becoming a dominant concern of the finance managers of all hues and shades.

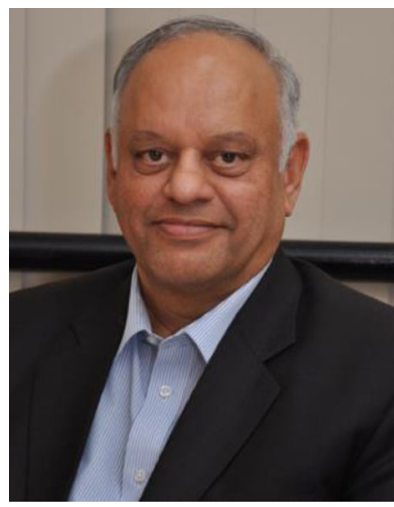

Chief Editor: $S$ V Raghavan

\footnotetext{
S. V. Raghavan

sv.raghavan@gov.in

1 Chennai, India
}

This issue is focusing on the related and interconnected issues highlighting the methods, practices, and successes, giving rise to what is today known broadly as FinTech or Financial Technologies. One gets news about institutions that are in core areas that are funded by the State in good measure; to some extent the corporate world; but one often does not get to see India and its part in the Globe in its entirety. In this June 2021 issue, readers will get a comprehensive and coherent picture of experiences in some of these. As we are moving the journal in to era of open exploration of complex usage of the Information and Communication Technology; the reliability and resilience of the ICT based systems have become important in the changed scenario, especially post Covid. When intense usage comes from unexpected quarters, the ICT designer and the Policy Gurus face the problem of complexity of the system coupled with the simplicity expected by the user, which one can call Designers' Dilemma. Well, what we have is just the beginning and perhaps the tip of the iceberg. In my usual style, it is my pleasure to introduce briefly the five articles of this issue.

1. The article by Shri Satish Babu and K M Abraham on 'Central bank digital currencies: policy and operational perspectives for India' introduces Cryptocurrencies and recounts their relationship to computing, networking. They trace the idea of an incentive regime to create secure, decentralized, peer-to-peer digital currency that lends itself to be used as a medium of exchange and as storage of value. While most Central Banks have been reluctant to permit cryptocurrencies considering risks to the financial system, they argue in favour of a Central Bank Digital Currency (CBDC) or 'Digital Fiat' that shares several advantages of 
cryptocurrencies but are entirely mandated and managed by the Central Bank. This paper examines and provides a background to CBDCs and discusses some of the policy and operational aspects that an Indian CBDC should consider.

2. The article 'Toward automated regulatory compliance' by Vinay Kulkarni et al., dwell upon the fact that businesses are getting increasingly regulated. As regulatory compliance is a board level concern and one of the top-3 CEO level concerns across business verticals, failure to do so leads not only to heavy fines but also avoidable reputational risk. The authors argue that the current practice of regulatory compliance is document-centric, and therefore, heavily reliant on human experts. Besides, they point out that given the large size of modern enterprises, their multi-geography operation, increasing dynamics, and frequent changes in regulations, the current practice of regulatory compliance is found wanting on correctness, responsiveness, and scale. The authors recommend introduction of appropriate technology to overcome these challenges and present an AI-aided model-driven automated approach to regulatory compliance and associated technology infrastructure. The highlight of the work is the description of how the approach suggested by the authors has fared in real-world industry-scale context.

3. The article, 'Emerging Insights from Digital Solutions in Financial Inclusion' by Sharon Buteau, Preethi Rao, and Fabrizio Valenti posit Digital Financial Services (DFS) hold the promise of bringing financial inclusion to the last mile. The authors emphasize that through the use of DFS, vulnerable and marginalized groups can reduce the risk and cost of using cash, and increase their access to a suite of financial services that would have been otherwise unavailable, or difficult to obtain, such as credit, savings, insurance, and pension. They explore the policy environment regulating digital finance in India as well as present high-level statistics on the level of digitization across population segments in the country. Finally the authors recommended developing customized digital financial services that address the unique needs and profiles of vulnerable groups, such as those specifically analysed in this paper.

4. The article on 'Behaviour science led technology for financial wellness' authored by Jayasree Raveendran, John Soren, Ramanathan V, Sudharshan R, Suman Mahalanabis, Suresh AK, and Vivek Balaraman, define Financial wellbeing is defined as having financial security and freedom of choice, in the present and in the future. Authors believe that this has a direct correlation to overall wellbeing: including productivity experienced at work, quality of relationship, quality of health and hence quality of life. The prevalence of concerns on financial wellness in fact, is witnessed across the globe. They argue that an approach to ameliorating this is through the field of behavioural finance which has uncovered many behavioural biases and barriers which impact how financial decisions are taken. Examples include lack of awareness of financial needs or awareness of the right products, lack of selfcontrol, confusion in making financial choices or being unable to save/invest at the right time. Such a solution helps users to visualize their financial needs, set financial goals and receive appropriate personalized nudges to help them in implementing financial plans as they go through various phases of career life stages.

5. The paper on 'Digital Financial Inclusion: Next Frontiers - Challenges and Opportunities' Chandra Mohan Malladi, Rupesh K. Soni, and Sanjay Srinivasan, addresses India's Financial Inclusion journey that is phenomenal in the last decade and its promotion by the Government of India through their Digital India Movement \& Prime Minister Jan Dhan Yojana (PM JDY). Reduction of poverty \& addressing the challenges of ensuring sustainable income could become a key factor to achieve an inclusive society. Information \& Communication Technology (ICT) are providing access to unbanked population progressively and helping to bring them into the banking segment. Digital Technologies are driving usage and making a positive impact on livelihood of citizens. In this paper we are discussing on what is achieved in Financial Inclusion (FI) so far and what next and how do we leverage and harness digital technologies to achieve an inclusive society. This paper enlists various challenges that continue to prevail in achieving an inclusive society and puts forth recommendations on addressing the key challenges and qualified the importance of collaboration and transparency between all the key stakeholders to achieve an inclusive ecosystem.

I would summarise the current issue as a bold attempt at presenting a few outstanding examples to the Government of India, rooted in practice and technology, discovering the vast usage potential and indigenous creativity potential spanning the June 2021 issue of CSI Transactions on ICT.

Happy Reading.

Professor S V Raghavan

Chief Editor 